\title{
Infrared divergences and non-lightlike eikonal lines in Sudakov processes
}

\author{
J.C. Collins, F. Hautmann \\ Department of Physics, Pennsylvania State University, University Park, PA 16802, USA \\ Received 3 September 1999; received in revised form 22 November 1999; accepted 22 November 1999 \\ Editor: H. Georgi
}

\begin{abstract}
We propose a method to analyze infrared contributions to non-inclusive processes in QCD. We use the one-loop Sudakov form factor as a working example. Borrowing techniques from renormalization theory, we construct counterterms for the contributions from the soft and collinear regions, and we relate them to eikonal Wilson lines taken along non-lightlike directions. (c) 2000 Published by Elsevier Science B.V. All rights reserved.
\end{abstract}

\section{Introduction}

Understanding the structure of hadronic final states in high energy collisions involves applying QCD in situations with multiple mass scales. While the application of QCD to inclusive hard-scattering processes characterized by a single large mass scale is well under control, the use of QCD in less inclusive, multiple-scale processes is much subtler [1].

A common feature of these more general situations is that soft gluon effects do not cancel. In tractable cases they merely factorize, and result in a more general factorization theorem, with new kinds of sensitivity to non-perturbative physics. An example is the transverse momentum distribution for the Drell-Yan process [2]. But we would like to treat, for example, Monte-Carlo event generators, where the theory does not yet [3] go systematically beyond the leading logarithm approximation, despite the great practical importance of these event generators. The necessary extensions of the factorization theorems require that we devise new more precise methods. This is the aim of the present paper.

We consider the well-known example of the one-loop part of the Sudakov form factor, Fig. 1a. A full factorization theorem was obtained in Ref. [4]. Our method is a systematic subtraction method that generalizes the Bogoliubov $R$-operation for renormalization. (For a related approach see Ref. [5].) The subtractions are obtained from matrix elements of certain gauge-invariant operators, which is essential if the factorization theorem is to be considered a real theorem of field theory. Moreover the subtractions are performed on the integrand, so that our final result for the ultraviolet part of the graph is infrared safe: it can be numerically integrated. Although our analysis is at the one loop level, the method should be generalizable to higher orders. 


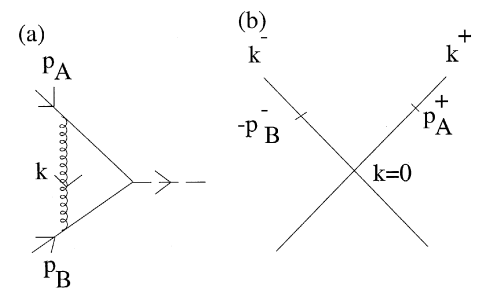

Fig. 1. (a) Form factor graph; (b) soft and collinear regions.

The plan of the paper is as follows. In Section 2 we describe the method and the form of the counterterms, which are obtained from matrix elements of path-ordered exponentials of the gluon field along non-lightlike lines. In Section 3 we discuss the evolution due to changes in these directions. In Section 4 we summarize and briefly discuss results and developments.

\section{The infrared subtraction method}

Consider the graph in Fig. 1a. To simplify the calculations while retaining all the essential ingredients on which we want to focus, we work in a massive abelian theory with scalar quarks. We denote the quark mass by $m$ and the gauge boson mass by $m_{g}$. We work in a center-of-mass frame in which the incoming quark momenta $p_{A}$ and $p_{B}$ are in the $+z$ and $-z$ directions, with $2 p_{A}^{+} p_{B}^{-}=Q^{2}$. We will compute the asymptotic large $Q$ behavior of the graph when the integrand is multiplied by a test function $\varphi(k / Q)$ :

$$
\begin{aligned}
\Gamma[\varphi]= & i g^{2} \int \frac{d^{4} k}{(2 \pi)^{4}} \frac{\left(2 p_{A}-k\right) \cdot\left(2 p_{B}+k\right)}{\left(k^{2}-m_{g}^{2}+i \varepsilon\right)\left[\left(p_{A}-k\right)^{2}-m^{2}+i \varepsilon\right]\left[\left(p_{B}+k\right)^{2}-m^{2}+i \varepsilon\right]} \varphi(k / Q) \\
& -\overline{\mathrm{MS}} \text { counterterm for UV divergence. }
\end{aligned}
$$

For the actual graph of Fig. 1a we will have $\varphi=1$. But retaining $\varphi(k / Q)$ until the end of the calculation will assist us in identifying the nature of the terms we obtain. It will also enable us to immediately solve a more general class of problem, when an infrared finite vertex correction is inserted.

Following the analysis of $[4,6,7]$ we identify the important regions of momentum space in $\Gamma$, as depicted in Fig. 1b:

1. soft region: all components of $k^{\mu}$ are much smaller than $Q: k^{\mu} \sim \lambda Q$, where $\lambda$ is small;

2. $p_{A}$-collinear region: $k^{+} \sim Q, k_{\perp} \sim \lambda Q, k^{-} \sim \lambda^{2} Q$, with $0 \leq k^{+} \leq p_{A}^{+}$;

3. $p_{B}$-collinear region: $k^{-} \sim Q, k_{\perp} \sim \lambda Q, k^{+} \sim \lambda^{2} Q$, with $-p_{B}^{-} \leq k^{-} \leq 0$;

4. hard region: all components of $k^{\mu}$ are of order $Q: k^{\mu} \sim Q$.

We will construct a term for each region. We will do this starting from the smallest region (region 1, representing a 0-dimensional subspace) and systematically going to larger regions: regions 2 and 3 (1-dimensional) and region 4 (4-dimensional). The strategy for constructing the approximation for a given region will be

a) take the original graph and subtract the terms for smaller regions;

b) construct an approximation valid in the region being treated;

c) show that it has no divergences or leading contributions from smaller regions;

d) "renormalize" divergences associated with larger regions.

Since, as we will see, only regions that are larger or smaller than the current region are relevant, the sum of all the terms will give a good leading-power approximation to the original graph. 


\subsection{Soft region}

We begin with the soft region. Expanding the integrand of $\Gamma$ in powers of the small parameter $\lambda$ (in the definition of the region) gives

$$
S_{0}=\frac{-i g^{2}}{(2 \pi)^{4}} \int d k^{+} d k^{-} d^{2} \boldsymbol{k} \frac{1}{\left(k^{2}-m_{g}^{2}+i \varepsilon\right)} \frac{1}{\left(k^{-}-i \varepsilon\right)\left(k^{+}+i \varepsilon\right)} \varphi(0) .
$$

The expansion in $\lambda$ gives the correct answer in the soft region unless one or both of the components $k^{+}, k^{-}$is too small. In that case $k^{+} k^{-} \ll \boldsymbol{k}^{2}$, and only the quark propagators have significant dependence on $k^{+}$and $k^{-}$. For the original graph, we deform the $k^{+}$and $k^{-}$contours out of the dangerous region. The approximation is only valid on the deformed contour, so that we must apply the same $i \varepsilon$ 's to the approximation in Eq. (2). Observe that the direction of the deformation of the contour integrations is determined by the fact that the interactions are initial-state interactions. Therefore the same deformation applies in all higher-order graphs as well.

Now we observe that Eq. (2) is the one-loop contribution to the vacuum expectation value of two Wilson lines $[4,7]$ taken along light-like directions $\hat{p}_{A}, \hat{p}_{B}$ :

$$
\tilde{S}_{0}=\frac{\left\langle 0\left|T\left[V_{q}\left(\hat{p}_{A}\right) V_{\bar{q}}\left(\hat{p}_{B}\right)\right]\right| 0\right\rangle}{\left\langle 0\left|V_{q}\left(\hat{p}_{A}\right)\right| 0\right\rangle\left\langle 0\left|V_{\bar{q}}\left(\hat{p}_{B}\right)\right| 0\right\rangle},
$$

where self-energy contributions have been divided out and we have defined

$$
V_{q}(n)=\mathscr{P} \exp \left(i g \int_{-\infty}^{0} d z A(z n) \cdot n\right), V_{\bar{q}}(n)=\mathscr{P} \exp \left(-i g \int_{-\infty}^{0} d z A(z n) \cdot n\right) .
$$

Our soft term $S_{0}$ still has singularities from the collinear and ultraviolet regions. The ultraviolet singularity is to be dealt with by the standard subtractive renormalization procedure, by adding a counterterm. We will now treat the collinear singularities in a similar fashion. The collinear singularities are entirely associated with the integration over the rapidity $y=\ln \left(k^{+} / k^{-}\right)$and are not removed by dimensional regularization [8].

As usual, the definition of the counterterms is non-unique. The reasons for our choice of the definition of the collinear counterterms are as follows. First, the new term is to have an operator interpretation, so that we can give it a nonperturbative definition. Secondly, the evolution equation is to be "simple", in a sense to be specified later. Here we just observe that the new definition will involve considering Wilson lines along an arbitrary direction, and the evolution equation treats the dependence on this direction.

Let us introduce two non-lightlike vectors $u_{A}, u_{B}: u_{A}=\left(u_{A}^{+}, u_{A}^{-}, \mathbf{0}\right), u_{B}=\left(u_{B}^{+}, u_{B}^{-}, \mathbf{0}\right)$. Then we define the subtracted soft term by

$$
\begin{aligned}
S= & \frac{-i g^{2}}{(2 \pi)^{4}} \int d k^{+} d k^{-} d^{2} \boldsymbol{k} \frac{1}{\left(k^{2}-m_{g}^{2}+i \varepsilon\right)}\left[\frac{1}{\left(k^{-}-i \varepsilon\right)\left(k^{+}+i \varepsilon\right)}\right. \\
& \left.-\frac{1}{\left(k^{-}-i \varepsilon\right)} \frac{u_{B}^{-}}{\left(u_{B}^{-} k^{+}+u_{B}^{+} k^{-}+i \varepsilon\right)}-\frac{u_{A}^{+}}{\left(u_{A}^{+} k^{-}+u_{A}^{-} k^{+}-i \varepsilon\right)} \frac{1}{\left(k^{+}+i \varepsilon\right)}\right] \varphi(0)-\mathrm{UV} .
\end{aligned}
$$

The first term in the square brackets is the unsubtracted soft term. The second term subtracts the divergence from the region collinear to $p_{A}$, i.e., from large positive rapidity $y$. The $u_{B}$ factor in this term provides a cut-off when the rapidity of $k$ goes much below the rapidity of $u_{B}$. Similarly, the third term subtracts the divergence from the region collinear to $p_{B}$. Observe that, in order to avoid invalidating the contour deformation argument, we must have $u_{A}^{+}, u_{B}^{-}>0, u_{A}^{-}, u_{B}^{+}<0$. Note that the collinear-to- $p_{A}$ subtraction term has no collinear-to- $p_{B}$ 
singularity; indeed it is power suppressed in this region. The same is true with $A$ and $B$ exchanged. Provided that we choose $u_{A}$ and $u_{B}$ to have large rapidities: $u_{A}^{+} / u_{A}^{-} \gtrsim p_{A}^{+} / p_{A}^{-}$, and $u_{B}^{+} / u_{B}^{-} \lesssim p_{B}^{+} / p_{B}^{-}$, then $S$ continues to provide a good approximation to $\Gamma$ in the soft region. The ultraviolet subtraction in the third line of Eq. (5) is understood to be done in the $\overline{\mathrm{MS}}$ scheme.

The cut-offs are gauge-invariant since, as we now show, they are obtained from a Wilson-line operators. Using the path-ordered exponentials introduced in Eq. (4), we define

$$
\tilde{S}=\frac{\left\langle 0\left|V_{q}\left(\hat{p}_{A}\right) V_{\bar{q}}\left(\hat{p}_{B}\right)\right| 0\right\rangle\left\langle 0\left|V_{q}\left(u_{A}\right)\right| 0\right\rangle\left\langle 0\left|V_{\bar{q}}\left(u_{B}\right)\right| 0\right\rangle}{\left\langle 0\left|V_{q}\left(\hat{p}_{A}\right) V_{\bar{q}}\left(u_{B}\right)\right| 0\right\rangle\left\langle 0\left|V_{q}\left(u_{A}\right) V_{\bar{q}}\left(\hat{p}_{B}\right)\right| 0\right\rangle},
$$

whose one-loop term gives Eq. (5). The first factor in the numerator gives the original soft term $S_{0}$ in Eq. (2), and the two factors in the denominator, involving eikonal lines along spacelike directions, give the collinear subtractions. The remaining factors in the numerator cancel factors of a complete external propagator for the Wilson line. Given our one-loop formulae, this result appears to us to be unique, if we simply assume that the quantity which we calculate is the product of vacuum expectation values of some Wilson line operators.

\subsection{Collinear to A region}

Next consider the collinear-to- $p_{A}$ region. We will construct a term $A$ such that $A+S$ gives a good approximation to $\Gamma$ (valid to the leading power) over the whole region, including its soft subregion. It is obtained by expanding $\Gamma-S$ in powers of the parameter $\lambda$ in the definition of the collinear-to- $p_{A}$ region, for which $k^{+} \sim Q, k_{\perp} \sim \lambda Q, k^{-} \sim \lambda^{2} Q$. Thus

$$
\begin{aligned}
A= & (\Gamma-S)_{\mid p_{A}-\text { coll. }}=\frac{i g^{2}}{(2 \pi)^{4}} \int d k^{+} d k^{-} d^{2} \boldsymbol{k} \frac{1}{\left(k^{2}-m_{g}^{2}+i \varepsilon\right)} \\
& \times\left[\frac{\left(2 p_{A}^{+}-k^{+}\right) 2 p_{B}^{-}}{\left(-2 p_{A}^{+} k^{-}+2 k^{+}\left[k^{-}-m^{2} /\left(2 p_{A}^{+}\right)\right]-\boldsymbol{k}^{2}+i \varepsilon\right)\left(2 p_{B}^{-} k^{+}+i \varepsilon\right)} \varphi\left(k^{+} / Q, 0, \mathbf{0}\right)\right. \\
& \left.+\frac{1}{\left(k^{-}-i \varepsilon\right)\left(k^{+}+i \varepsilon\right)} \varphi(0)-\frac{1}{\left(k^{-}-i \varepsilon\right)} \frac{u_{B}^{-}}{\left(u_{B}^{-} k^{+}+u_{B}^{+} k^{-}+i \varepsilon\right)} \varphi(0)\right]-\mathrm{UV} .
\end{aligned}
$$

By this construction of $A$, we ensure that $A+S$ is a good approximation to the collinear-to- $p_{A}$ region. As to the soft region, observe that the first term in square brackets, the collinear expansion of the original graph, has a soft singularity at $k^{+}=0$. But the second term, obtained from $S$, cancels this singularity. Notice that the third term is power suppressed in the soft region. Hence $A+S$ is a good approximation to the original graph in a neighborhood of both the soft and collinear-to- $p_{A}$ regions.

We now show that the $A$ term, Eq. (7), is power suppressed in region 3, where $k$ is collinear to $p_{B}$. This region merely overlaps with the region that this term is designed to treat. In the $p_{B}$-collinear region, a good approximation is obtained by replacing the quark factor $\left(2 p_{A}^{+}-k^{+}\right) /\left[\left(p_{A}-k\right)^{2}-m^{2}+i \varepsilon\right]$ by the light-like eikonal $2 p_{A}^{+} /\left(-2 p_{A}^{+} k^{+}-i \varepsilon\right)=-1 /\left(k^{+}+i \varepsilon\right)$. This is the same replacement as for the soft region, so that we get a suppression. This is a result and solves what is effectively a problem in overlapping divergences: We obtained $A$ by expanding about the line $k^{-}=\boldsymbol{k}_{T}=0-$ Fig. 1b, and it is suppressed at the intersection, $k=0$, with the collinear-to- $p_{B}$ line. The extrapolation back to the whole of the $B$ region continues to be suppressed.

We therefore have the following results:

- The sum $A+S$ gives a good approximation to $\Gamma$ in regions 1 and 2 .

- The term $A$ is power suppressed in regions that overlap with the $A$ region or are smaller than it. 


\subsection{Collinear to B region}

The expression for the $p_{B}$-collinear term, $B$, is simply obtained from Eq. (7) by interchanging the indices + and,$- A$ and $B$.

Just as for the soft term $S$, the collinear terms can be obtained uniquely as the one-loop expansion of a product of gauge-invariant matrix elements:

$$
\tilde{A}=\frac{\left\langle 0\left|V_{\bar{q}}\left(\hat{p}_{B}\right) q(0)\right| p_{A}\right\rangle\left\langle 0\left|V_{q}\left(\hat{p}_{A}\right) V_{\bar{q}}\left(u_{B}\right)\right| 0\right\rangle}{\left\langle 0\left|V_{q}\left(\hat{p}_{A}\right) V_{\bar{q}}\left(\hat{p}_{B}\right)\right| 0\right\rangle\left\langle 0\left|V_{\bar{q}}\left(u_{B}\right)\right| 0\right\rangle}, \quad \tilde{B}=\frac{\left\langle 0\left|\bar{q}(0) V_{q}\left(\hat{p}_{A}\right)\right| p_{B}\right\rangle\left\langle 0\left|V_{q}\left(u_{A}\right) V_{\bar{q}}\left(\hat{p}_{B}\right)\right| 0\right\rangle}{\left\langle 0\left|V_{q}\left(\hat{p}_{A}\right) V_{\bar{q}}\left(\hat{p}_{B}\right)\right| 0\right\rangle\left\langle 0\left|V_{q}\left(u_{A}\right)\right| 0\right\rangle} .
$$

\subsection{Ultraviolet region}

Finally, we consider the hard part of the form factor. We first observe that the sum of the terms for the smaller regions, $A+B+S$, provides a good approximation to $\Gamma$ in the whole of the smaller regions 1,2 , and 3. To obtain a term $H$ for the hard region (4), we simply take the massless approximation to $\Gamma-A-B-S$

$$
\begin{aligned}
H= & (\Gamma-S-A-B)_{\mid m, m_{g} \rightarrow 0}-\mathrm{UV} \\
= & \frac{i g^{2}}{(2 \pi)^{4}} \int d k^{+} d k^{-} d^{2} \boldsymbol{k} \frac{1}{\left(2 k^{+} k^{-}-\boldsymbol{k}^{2}+i \varepsilon\right)} \\
& \times\left[\frac{4 p_{A}^{+} p_{B}^{-}+2 p_{A}^{+} k^{-}-2 p_{B}^{-} k^{+}-2 k^{+} k^{-}+\boldsymbol{k}^{2}}{\left(-2 p_{A}^{+} k^{-}+2 k^{+} k^{-}-\boldsymbol{k}^{2}+i \varepsilon\right)\left(2 p_{B}^{-} k^{+}+2 k^{+} k^{-}-\boldsymbol{k}^{2}+i \varepsilon\right)} \varphi(k / Q)\right. \\
& -\frac{2 p_{A}^{+}-k^{+}}{\left(k^{-}-i \varepsilon\right)\left(k^{+}+i \varepsilon\right)} \varphi(0)-\frac{2 k^{-}}{\left(-2 p_{A}^{+} k^{-}+2 k^{+} k^{-}-\boldsymbol{k}^{2}+i \varepsilon\right)\left(k^{+}+i \varepsilon\right)} \varphi\left(k^{+} / Q, 0, \mathbf{0}\right) \\
& \left.+\frac{2 k^{-}}{\left(k^{-}-i \varepsilon\right)\left(2 p_{B}^{-} k^{+}+2 k^{+} k^{-}-\boldsymbol{k}^{2}+i \varepsilon\right)} \varphi\left(0, k^{-} / Q, \mathbf{0}\right)\right]-\mathrm{UV} .
\end{aligned}
$$

Note that the all non-lightlike eikonal terms have canceled.

To verify that the subtractions are correct, we perform the $k^{+}, k^{-}$integrations in the case $\varphi=1$. This gives

$$
H=\frac{-g^{2}}{8 \pi^{2}} \int \frac{d \boldsymbol{k}^{2}}{\boldsymbol{k}^{2}}\left\{\ln \left(\frac{\boldsymbol{k}^{2}}{Q^{2}}\right)+i \pi+\frac{1-\boldsymbol{k}^{2} / Q^{2}}{R}\left[\ln \left(\frac{1+R}{1-R}\right)-i \pi\right]\right\}-\mathrm{UV}
$$

where $Q^{2}=2 p_{A}^{+} p_{B}^{-}$and

$$
R= \begin{cases}\sqrt{1-4 \boldsymbol{k}^{2} / Q^{2}} & \text { if } 4 \boldsymbol{k}^{2} / Q^{2} \leq 1, \\ i \sqrt{4 \boldsymbol{k}^{2} / Q^{2}-1} & \text { if } 4 \boldsymbol{k}^{2} / Q^{2} \geq 1\end{cases}
$$

Observe that, as a result of the infrared subtractions, the $\boldsymbol{k}^{2}$ integration in Eq. (10) is regular at small $\boldsymbol{k}^{2}$. The large $\boldsymbol{k}^{2}$ behavior, on the other hand, has to be dealt with via an ultraviolet subtraction.

Eqs. (5), (7) and (9) provide the main result of the application of our method. The analysis of Refs. [4,6,7] establishes the list of leading regions. For each region, we have defined a term that, added to the terms for smaller regions, gives a good leading-power approximation to the original graph in that region. For each term, we have shown that it does not receive leading contributions from regions that are smaller or have an overlap 
with the region being considered. Thus the sum of all the terms gives a good leading-power approximation to the original graph:

$$
H+A+B+S=\Gamma+\text { nonleading power. }
$$

\section{Evolution}

The spacelike vectors $u_{A}, u_{B}$ in the soft and collinear subtractions are not physical parameters, and the sum $H+A+B+S$ is independent of them. The utility of these vectors comes from the fact that evolution equations in $y_{A}=\frac{1}{2} \ln \left|u_{A}^{+} / u_{A}^{-}\right|$and $y_{B}=\frac{1}{2} \ln \left|u_{B}^{+} / u_{B}^{-}\right|$can be applied to the factors in the factorization formula to extract effects associated with large logarithms [2,4]. For example, differentiating Eq. (5) with respect to $y_{A}$ gives

$$
\frac{\partial S}{\partial y_{A}}=\frac{i g^{2}}{(2 \pi)^{4}} \int \frac{d^{4} k}{\left(k^{2}-m_{g}^{2}+i \varepsilon\right)} \frac{u_{A}^{2}}{\left(u_{A}^{+} k^{-}+u_{A}^{-} k^{+}-i \varepsilon\right)^{2}} .
$$

This is to be contrasted with the evolution equations obtained in Ref. [4], which suffer from power-law corrections. Our new method has all the power law corrections in the initial construction of the $A, B, S$ and $H$ terms. Since no further approximations are needed in solving the evolution equations [5], this can make for easier and cleaner proofs in more complicated situations. It also makes it easier to locate the sources of the power-law corrections: they are all associated with the approximations made to derive the factorization.

\section{Outlook}

In this paper we have proposed a systematic subtraction procedure to analyze infrared contributions to non-inclusive scattering processes in QCD. The counterterms arise from gauge-invariant Wilson line operators which are in spacelike directions. The form of these operators allows simple evolution equations to be obtained, which have no power-law corrections; this makes it much easier to pinpoint where the power-law errors in factorization theorems occur. Observe that even in our one-loop example, the pattern of infrared contributions in the collinear regions is equivalent to that of overlapping divergences in renormalization theory.

Uniqueness of the operator formulae follows from the assumption of a factorized form for the complete amplitudes. Given the general and systematic nature of our approach, we expect to generalize it to all higher orders and to other, more physically interesting, processes.

\section{Acknowledgements}

This research is supported in part by the US Department of Energy under grant No. DE-FG02-90ER-40577.

\section{References}

[1] See, for instance, G. Sterman, in: D.E. Soper (Ed.), QCD and beyond, Proceedings of the Theoretical Advanced Study Institute TASI'95, World Scientific, Singapore, 1996, p.327.

[2] J.C. Collins, D.E. Soper, G. Sterman, Nucl. Phys. B 250 (1985) 199.

[3] For recent studies in this direction, see C. Friberg, T. Sjöstrand, hep-ph/9906316; S. Mrenna, hep-ph/9902471.

[4] J.C. Collins, in: A.H. Mueller (Ed.), Perturbative Quantum Chromodynamics, World Scientific, Singapore, 1989 , p.573.

[5] F.V. Tkachov, Int. J. Mod. Phys. A 8 (1993) 2047; F.V. Tkachov, hep-ph/9703423.

[6] S.B. Libby, G. Sterman, Phys. Rev. D 18 (1978) 3252; ibid. 18 (1978) 4737.

[7] G.P. Korchemsky, A.V. Radyushkin, Sov. J. Nucl. Phys. 45 (1987) 910.

[8] J.C. Collins, F.V. Tkachov, Phys. Lett. B 294 (1992) 403. 